\title{
b) Extremitäten
}

\section{Einleitung}

\author{
G. M. Lösch
}

Klinik für Plastische und Wiederherstellungschirurgie, Klinikum der Medizinischen Hochschule, Ratzeburger Allee 160, D-2400 Lübeck

Einen Hinweis auf die Bedeutung einer optimalen primären Versorgung von Verletzungen der Extremitäten ergibt sich aus statistischen Angaben über deren Häufigkeit.

Aus der Unfallanalyse von Abt (1982) ${ }^{1}$ geht hervor, daß im Jahre 1980 von den gewerblichen Berufsgenossenschaften in der Bundesrepublik Deutschland insgesamt 1702506 Unfälle registriert wurden, dabei handelte es sich in $21,6 \%$ der Fälle um Verletzungen der Hände, in 16,1\% der Füße und in $9,2 \%$ der Kniegelenke und Unterschenkel.

Bei den statistischen Untersuchungen von 100 Fällen von Handverletzungen, die zur primären Behandlung in unsere Klinik überwiesen worden waren, lagen bei $40 \%$ Hautdefekte vor, die eine primäre Wiederherstellung durch freie oder gestielte Hauttransplantation benötigten.

Wir möchten nun Herrn Lanz um sein Referat über ,Indikation und Operationstechnik bei Fingerkuppendefekten" bitten.

$1 \mathrm{~W}$. Abt: Unfallanalyse „80“. Schriftenreihe des Hauptverbandes der gewerblichen Berufsgenossenschaften e.V. Hrsg. Hauptverband der gewerblichen Berufsgenossenschaften, Langwartweg 103, D-5300 Bonn 1, 1980 